\section{Cycle cellulaire et prolifération}

L'article de Darius Wolowiec et Martine Ffrench (p. 165 de ce numéro) présente différents exemples de l'intervention des kinases liées au cycle cellulaire et aux cyclines dans les cancers humains. Ces kinases sont contrôlées par des inhibiteurs dont p1 ${ }^{\mathrm{INK}}$, codé par le locus MTS1/CDKN2 qui semble fréquemment modifié dans les tumeurs humaines, leucémies et carcinomes. Le locus MTS1/CDKN2 a la stupéfiante caractéristique de coder pour deux protéines

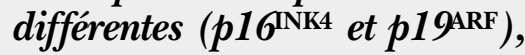
résultats de l'utilisation de promoteurs et d'exons alternatifs et de la lecture d'un exon commun dans deux phases différentes, protéines inhibant toutes deux le cycle cellulaire par deux mécanismes distincts. Des progrès ont été accomplis également dans l'identification des voies de transmission des signaux de prolifération, la voie Tiam/Rac s'ajoutant maintenant à la voie Sos/Ras connue depuis quelques années. Enfin, les expériences d'invalidation homo- ou hétérozygotes des allèles p53, $\mathrm{rb}$, et $\mathrm{mdm} 2$ permettent de mieux cerner le rôle de $p 53$ dans le contrôle du cycle cellulaire, la coopération avec $R \boldsymbol{R}$ et le partenariat avec Mdm2. Des résultats récents suggèrent que le rôle essentiel, vital, de Mdm2 est de régler négativement l'activité de p53.

\title{
Nouveaux modèles murins d'étude du gène suppresseur de tumeur p53
}

La technique de recombinaison homologue pour inactiver l'expression d'un gène est largement utilisée pour l'étude des oncogènes et des gènes suppresseurs de tumeur. A l'heure actuelle, le nombre de lignées de souris dont le génome a ainsi été modifié s'élève à plus de 263 si l'on se réfère à la récente compilation publiée dans Current Biology [1]. Les altérations obtenues sont extrêmement variables et peuvent aller de l'absence de tout phénotype, dans le cas de redondances fonctionnelles par exemple, à une létalité embryonnaire précoce, telle que cela a été observé dans le cas du gène de susceptibilité au rétinoblastome $(R b)$. Il est maintenant possible d'obtenir de nouvelles combinaisons d'altérations génétiques, plus propices à l'étude des maladies cancéreuses dont les origines sont multigéniques, en croisant les différents animaux déjà génétiquement manipulés (soit par inactivation d'un gène par recombinaison homologue, soit par surexpression d'un transgène intégré dans le génome de l'animal). Dans cette revue, nous nous intéresserons aux modèles murins mettant en jeu le gène suppresseur de tumeur p53, ses différents partenaires ainsi que divers gènes impliqués dans la transformation cellulaire.

Altération de la fonction du gène $p 53$ et transformation cellulaire

Le gène suppresseur de tumeur $p 53$ code pour une phosphoprotéine nucléaire dont la fonction est altérée, en général à la suite de mutations ponctuelles, dans plus de $50 \%$ des cancers humains. Il s'agit de l'altération génétique la plus fré- quemment observée, tous types de tumeurs confondues $\left(m / s n^{\circ} 2\right.$, vol. 4 , p. 122; $n^{\circ} 8$, vol. 5 , p. 598 ; $n^{\circ} 10$, vol. 5, p. 789; n०9, vol. 8 , p. 996) [2, 3]. En 1989, Lavigueur et al. (Toronto, ON, CA) décrivaient les premières souris transgéniques surexprimant une protéine p53 mutante, dont le clone génomique avait été isolé à partir de cellules murines érythroleucémiques transformées par le virus de Friend [4]. Ces animaux développent, en 15 à 18 mois, pour plus de $20 \%$ d'entre eux, des tumeurs de type adénomes pulmonaires, ostéosarcomes et lymphomes. Cette distribution particulière de tumeurs a permis d'établir une association entre l'altération du gène $p 53$ et le syndrome de Li-Fraumeni, maladie autosomique dominante caractérisée par l'apparition de cancers multiples, principalement de tumeurs du sein, d'ostéosarcomes et de lymphomes, et pour laquelle il existe une stricte corrélation entre la transmission de l'allèle muté et l'apparition du cancer $(\mathrm{m} / \mathrm{s}$ $n^{\circ} 7$, vol. 8, p. 733) [5]. En 1992, l'établissement de lignées de souris déficientes en gène $p 53$ par l'équipe de Donehower (Houston, TX, USA) a, de façon déterminante, confirmé l'incidence de la perte de fonction du gène $p 53$ sur l'apparition d'un phénotype tumoral $\left(\mathrm{m} / \mathrm{s} n^{\circ} 5\right.$, vol. 8 , p. 492) [6]. Ces animaux, qui ont un développement embryonnaire normal, meurent en 4 à 6 mois de divers types de cancers, majoritairement des lymphomes (plus récemment, des malformations crâniofaciales et oculaires ont été observées au cours du développement chez ces souris, le taux de ces 
anomalies variant avec le fond génétique de l'animal [7]). De façon intéressante, l'examen des tumeurs développées chez les animaux hétérozygotes $p 53+/-$ (qui apparaissent à un âge plus avancé) indique que l'allèle sauvage restant a été perdu au cours de la tumorigenèse, comme cela s'observe dans la plupart des cancers humains présentant des mutations du gène $p 53$.

La création de souris déficientes en gène $p 53 \mathrm{a}$, par la suite, fourni un matériel d'intérêt majeur pour l'étude des événements et/ou altérations génétiques permettant l'acquisition du caractère transformé. Ainsi récemment, Harvey et al. (Houston, TX, USA et Toronto, ON, CA) ont croisé les souris surexprimant des protéines p53 mutantes ( $p 53$ mut) avec des animaux présentant une inactivation de l'un ( $p 53+/-)$ ou des deux allèles ( $p 53-/-)$ de ce même gène [8]. Le phénotype le plus intéressant concerne les animaux p53+/-; p53mut (Tableau I) qui développent des cancers de nature plus variée et d'apparition plus rapide que les souris parentales $p 53+/-$ ou $p 53 m u t$. Ce résultat confirme l'implication in vivo de la présence d'une p53 mutante sur le processus de transformation cellulaire, dans un contexte de déficit partiel en gène suppresseur de tumeur $p 53$ (figure 1). Ainsi, l'expression d'une protéine p53 mutante apparaît constituer un événement dominant, dans la mesure où le phénotype du produit de l'allèle muté neutralise celui du produit de l'allèle normal restant. L'analyse ex vivo de la vitesse de croissance des fibroblastes embryonnaires issus des souris p53+/-; p53mut a, de plus, confirmé que ces cellules possédaient des propriétés de multiplication bien supérieures à celles des fibroblastes de souris $p 53+/-$. Cependant, le mécanisme de l'apparente dominance de p53 mutée sur le produit de l'allèle muté reste inconnu. La protéine p53 exerce ses fonctions au sein de la cellule sous une forme tétramérique et des hétéro-oligomères mixtes p53mut; p53 normale peuvent avoir perdu toute activité biologique. Il est aussi possible que certaines protéines p53 mutantes aient de nouvelles fonctions

\begin{tabular}{|c|c|}
\hline \multicolumn{2}{|r|}{ Tableau I } \\
\hline & Phénotype \\
\hline p53+/- & $\begin{array}{l}\text { viable } \\
\text { grande fréquence de lymphomes et de sarcomes } \\
50 \% \text { de mortalité à } 18 \text { mois }\end{array}$ \\
\hline p53-1- & $\begin{array}{l}\text { viable } \\
\text { grande fréquence de lymphomes et de sarcomes } \\
100 \% \text { de mortalité à } 11 \text { mois }\end{array}$ \\
\hline$R b 1+/-$ & $\begin{array}{l}\text { viable } \\
100 \% \text { d'adénome de I'hypophyse à } 10-15 \text { mois }\end{array}$ \\
\hline$R b 1-/-$ & Mort à 16 jours de vie embryonnaire \\
\hline p53 mut & $\begin{array}{l}\text { viable } \\
\text { lymphome et adénocarcinome } \\
30 \% \text { de mort à } 18 \text { mois }\end{array}$ \\
\hline$m d m 2+/-$ & viable \\
\hline$m d m 2-/-$ & mort à 5 jours de vie embryonnaire \\
\hline$W A F 1+/-$ & $\begin{array}{l}\text { viable } \\
\text { pas de cancer détectable }\end{array}$ \\
\hline WAF1-/- & $\begin{array}{l}\text { viable } \\
\text { pas de cancer; perte de la fonction d'arrêt en G1 } \\
\text { après irradiation des cellules }\end{array}$ \\
\hline p53+/-; p53mut & $\begin{array}{l}\text { viable } \\
\text { lymphomes, sarcomes et adénocarcinomes } \\
\text { bronchiques } \\
\text { apparition précoce de cancer } \\
70 \% \text { de mort à } 18 \text { mois }\end{array}$ \\
\hline p53-1-; p53mut & $\begin{array}{l}\text { viable } \\
\text { grande fréquence de lymphomes et de sarcomes } \\
100 \% \text { de mortalité à } 11 \text { mois }\end{array}$ \\
\hline$p 53+/-; R b+/-$ & $\begin{array}{l}\text { viable } \\
\text { nombreux cancers médullaires de la thyroïde } \\
\text { et de I'hypophyse } \\
100 \% \text { de mort à } 16 \text { mois }\end{array}$ \\
\hline$p 53-1-; R b+/-$ & $\begin{array}{l}\text { viable } \\
\text { nombreux cancers médullaires de la thyroïde, } \\
\text { de I'hypophyse et de lymphomes } \\
100 \% \text { de mort à } 6 \text { mois }\end{array}$ \\
\hline CD2-myc & $\begin{array}{l}\text { viable } \\
20 \% \text { de lymphomes T entre } 3 \text { et } 12 \text { mois }\end{array}$ \\
\hline p53-1-; CD2-myc & $\begin{array}{l}\text { viable } \\
100 \% \text { de lymphomes T en } 2 \text { mois }\end{array}$ \\
\hline MMTV-myc & $\begin{array}{l}\text { viable } \\
\text { carcinomes mammaires chez les femelles } \\
\text { en } 8-10 \text { mois }\end{array}$ \\
\hline MMTV-myc; p53+/- & $\begin{array}{l}\text { viable } \\
\text { lymphomes, en } 4 \text { mois chez les femelles, } \\
\text { et en } 6 \text { mois chez les mâles }\end{array}$ \\
\hline
\end{tabular}


En 1991, David Lane a proposé un modèle selon lequel la protéine p53 serait impliquée dans le contrôle de l'intégrité génétique des cellules [9]. Les diverses fonctions qu'exerce cette protéine au sein de la cellule (activation de gènes impliqués dans l'arrêt de la division cellulaire et de la réplication, dans la réparation de l'ADN et, sous certaines conditions, dans la mise en place d'un processus d'apoptose) peuvent en effet rendre compte d'une instabilité génétique importante en cas d'absence de protéine p53 fonctionnelle (voir figure 2). Afin de tester le taux de mutations endogènes présentes chez les souris déficientes en protéine p53, deux équipes ont eu l'idée de croiser ces animaux avec des souris BigBlue $[10,11]$. Ces dernières possèdent un transgène correspondant au gène lac I (répresseur de l'opéron lactose) encadré par des séquences Cos du bactériophage lambda [12]. En absence de mutation, l'encapsidation in vitro de l'ADN de ces souris et leur intro-

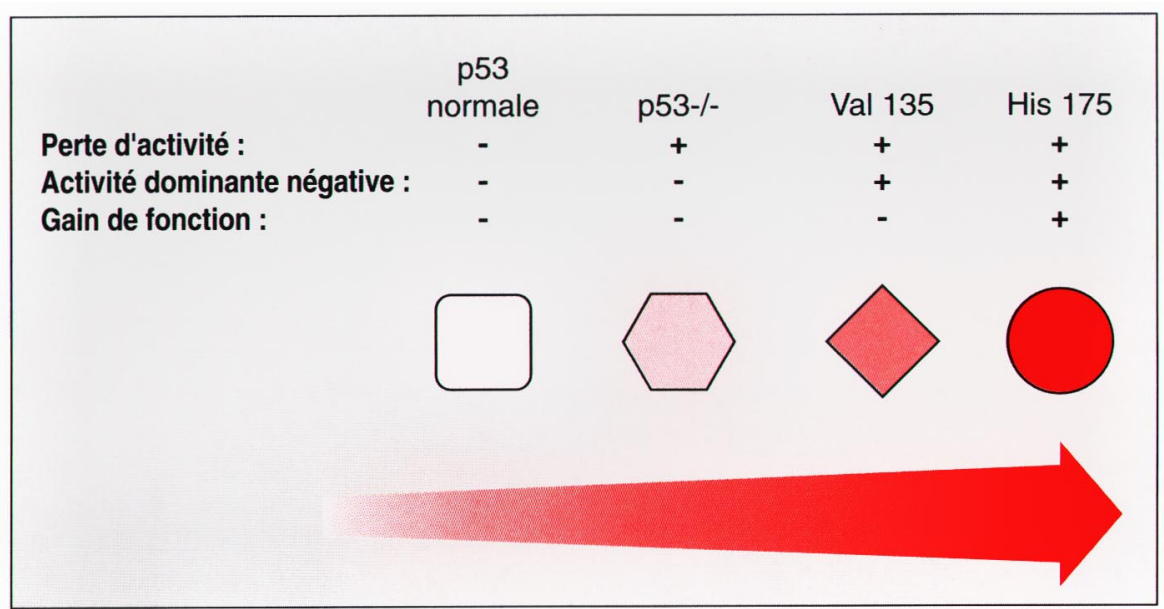

Figure 1. Hétérogénéité des mutants p53. Plus de 100 codons du gène p53 peuvent être la cible de mutations. Cette très grande diversité conduit à l'obtention de mutants pouvant avoir des propriétés différentes. A l'heure actuelle, on définit trois classes de mutants $\mathrm{p} 53: 1$ ) les mutations nulles conduisant à une p53 totalement inactive n'intervenant pas directement dans la transformation (par exemple, souris déficientes en p53); 2) les mutations dominantes négatives conduisant à une p53 totalement inactive mais restant capable d'interférer avec la p53 exprimée par l'allèle sauvage (ex.: mutant p53 $\mathrm{Val}^{135}$ ) et 3 ) les mutations dominantes positives conduisant à une $p 53$ totalement inactive. De plus, la p53 mutante gagne un pouvoir oncogène qui intervient directement dans la transformation (ex. p53His ${ }^{175}$ ).

Figure 2. p53 et ses divers partenaires. La p53 est capable de régler l'expression de plusieurs gènes dont certains, comme WAF-1, BAX ou IGF-BP3 (insulin-like growth factor binding protein) sont reliés à l'activité apoptotique ou antiproliférative de p53. En revanche, la raison pour laquelle des gènes tels que cycline G et TGF- $\alpha$ sont activés par p53 reste

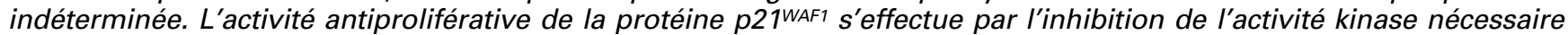

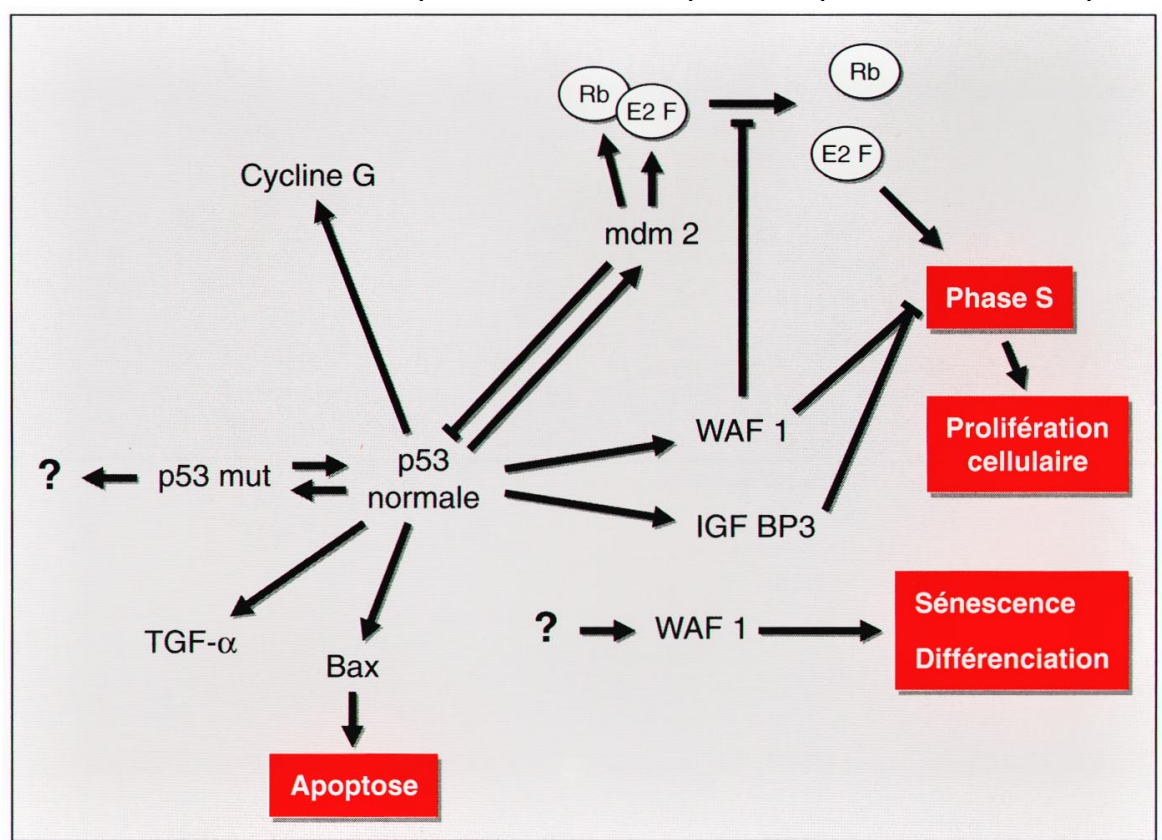

$m / s n^{\circ} 2$, vol. 12 , féurier 96 à la phosphorylation de la protéine $R b$ et la libération des facteurs E2F. La protéine p21WAF1 est aussi impliquée dans la différenciation et la sénescence par des mécanismes ne faisant pas appel à la p53. La p53 active la transcription du gène mdm2 qui, en retour, inhibe l'activité de la p53. Ce phénomène de rétrocontrôle servirait à moduler l'activité de la p53. Récemment, des interactions entre $m d m-2 / E 2 F$ et $m d m-2 / R b$ ont été mises en évidence [31, 32]. Ces interactions induiraient la libération du facteur de transcription E2F et la stimulation de gènes impliqués dans la transition vers la phase $S$ suggérant que $\mathrm{mdm} 2$ aurait un rôle directeur sur la régulation du cycle cellulaire. Les lignes terminées par une pointe de flèche marquent les interactions positives alors que celles terminées par une barre perpendiculaire marquent les interactions négatives. 
duction dans une bactérie indicatrice engendrent des clones blancs. En cas de mutations touchant le gène lac I, les plages sont bleues. Ces souris ont notamment été utilisées dans des études de spécificité tissulaire de certains carcinogènes, l'intérêt d'une telle approche résidant dans le fait que l'ADN peut être extrait de n'importe quel tissu de l'animal. Ainsi, des souris BigBlue traitées avec de la méthylnitroso-urée engendrent des clones bleus lorsque la banque est faite à partir d'ADN de rate, mais pas à partir de cerveau ou de foie [12]. Lorsque l'on examine la fréquence de clones bleus des souris BigBlue normales ou BigBlue; p53-/-, aucune différence n'est observée et ce quel que soit le tissu analysé $[10,11]$. Ce résultat, apparemment surprenant, indique que le taux de mutations est identique chez les souris normales ou p53-/- (on ne discute ici que des altérations visibles par cette approche, c'est-à-dire mutations faux sens, non sens ou petits réarrangements) et donc que les tumeurs développées par les souris p53-/- ne sont pas la conséquence d'une accumulation de mutations ponctuelles. L'hypothèse retenue ici est que la protéine p53 pourrait être impliquée dans la stabilité génétique au sens global du terme (aneuploïdie, amplification génique ou réarrangements chromosomiques) et non pas, comme proposé précédemment, dans la réparation des mutations ponctuelles.

\section{Altération de la fonction des gènes WAF-1 et MDM2, partenaires de la p53}

- p53, WAF-1 et contrôle du cycle cellulaire

Le gène $W A F-1 / C I P-1$, l'un des gènes cibles de la protéine p53, code pour une protéine p21 WAF-1/CIP-1 inhibitrice de l'activité kinase des complexes cdk/cyclines et plus particulièrement du complexe cdk2/cycline $\mathrm{E}$ nécessaire à la transition G1/S du cycle cellulaire $(\mathrm{m} / \mathrm{s}$ $n^{\circ} 6-7$, vol. 10, p. 744) [13, 14]. Le produit du gène WAF-1 a aussi été impliqué dans d'autres processus indépendants de p53 tels que la sénescence ou la différenciation nelle du gène WAF-1 par la protéine p53 (nulle dans le cas de protéines p53 mutantes) a fourni, jusqu'à très récemment, un modèle simple rendant compte du rôle de la p53 dans l'arrêt de la division cellulaire (figure 2) [13]. A première vue, ce modèle est conforté par l'analyse de lignées de souris déficientes en gène WAF-1 [18] pour lesquelles il a été montré que des cellules fibroblastiques avaient perdu leur capacité d'arrêter le cycle cellulaire en phase G1 après lésion de l'ADN. Néanmoins, l'absence de phénotype visible chez les souris WAF-1-/-, qui ne présentent aucune prédisposition à développer à moyen terme (7 mois de recul) des tumeurs, apparaît paradoxale au vu de ce modèle.

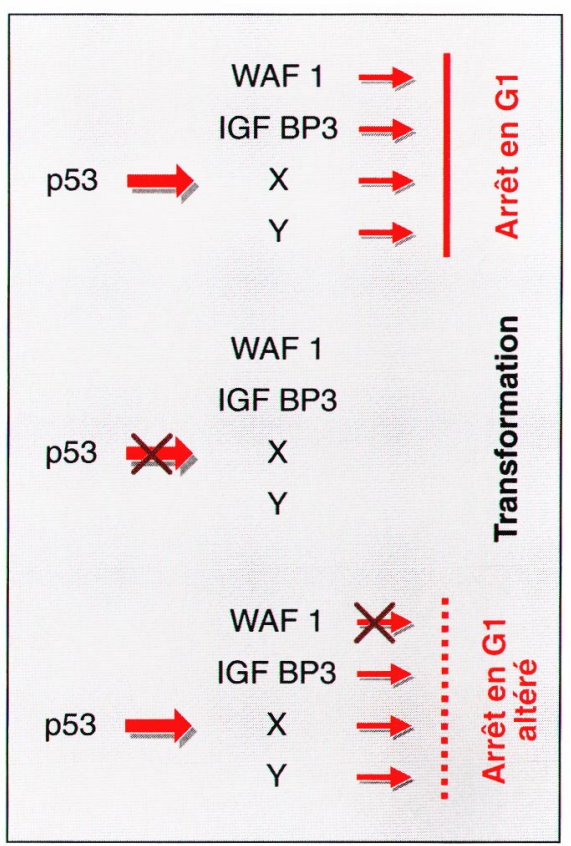

Figure 3. Activité antiproliférative de p53. L'arrêt en phase G1 induit par p53 semble nécessiter plusieurs médiateurs dont WAF-1, IGF-BP3 ou les produits d'autres gènes qui restent à identifier. L'inactivation de p53, en altérant l'ensemble de ces voies, serait capable d'induire la transformation cellulaire tandis que l'inactivation d'un seul médiateur n'aurait qu'un effet atténué.
Pour expliquer cette apparente contradiction, les auteurs soulignent le fait que la perte de fonction des cellules fibroblastiques WAF-1-/-reste cependant moins drastique que dans le cas des fibroblastes $p 53-/-$. Il y aurait donc, au sein de la cellule, plusieurs voies de régulation négative du cycle cellulaire dépendantes de p53, dont certaines ne nécessiteraient pas la présence de la protéine p21 ${ }^{\text {CIP1/WAF1 }}$ (figure 3). Cette hypothèse a été récemment confortée par la caractérisation d'un nouveau gène cible de la protéine $\mathrm{p} 53$, le gène $I G F$ BP3 (insulin-like growth factor binding protein), dont le produit est impliqué dans la régulation négative du cycle cellulaire [19].

- p53, MDM2 et transformation cellulaire

Il existe une double régulation entre les produits des gènes $p 53$ et $M D M 2$ : et leurs produits: l'expression du gène MDM2 est stimulée par la protéine p53 [20] et, d'autre part, la protéine Mdm2 interagit spécifiquement avec le domaine de transactivation de la protéine p53 et inhibe son activité $\left(\mathrm{m} / \mathrm{s} n^{\circ} 8-9\right.$, vol. 9 , p. 998; $n^{\circ} 8$, vol. 11, p. 1185). Cette régulation croisée entre p53 et Mdm2 est supposée faire partie de l'un des systèmes réglant temporellement l'activité de la protéine p53 après un stress génotoxique. On connaît peu de chose encore sur le produit du gène $M D M 2$. La régulation de son expression est relativement complexe du fait de la présence de plusieurs transcrits traduits en au moins trois formes de protéines. La structure primaire des protéines MDM2 suggère qu'elles pourraient être des facteurs de transcription dont, cependant, les gènes cibles n'ont pas été caractérisés. Le gène MDM2 est fréquemment amplifié dans les sarcomes des tissus mous dans lesquels, dans la majorité des cas, les mutations du gène $p 53$ sont absentes. En 1995, deux laboratoires ont établi des lignées de souris déficientes en gène $m d m 2$. Alors que les souris $m d m 2+/-$ sont viables et ne présentent aucun phénotype particulier, les animaux homozygotes $m d m 2-/-$ meurent in utero à cinq jours post-coitum [21, 22]. L'analyse des blastocystes n'a pas 


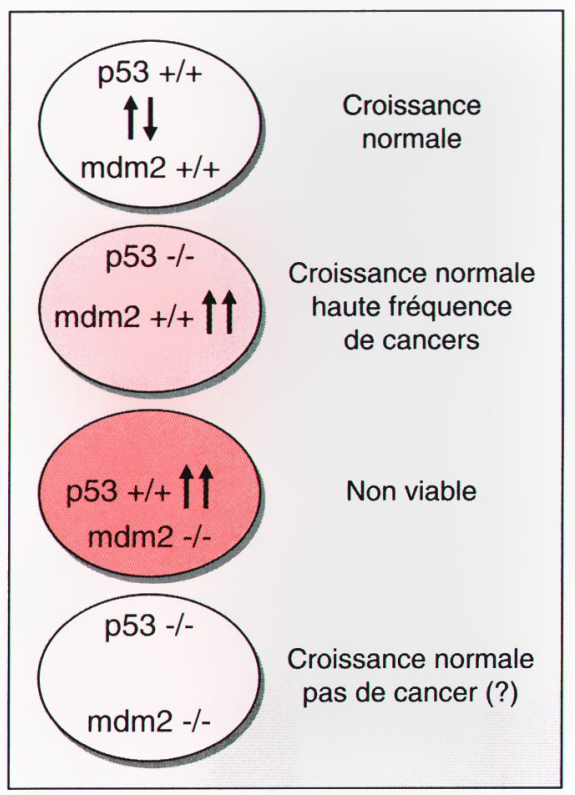

Figure 4. Dialogue entre p53 et Mdm2. Dans une cellule normale, il existe un équilibre entre l'acitvité de la p53 et mdm2. En l'absence de mdm2, la synthèse de p53 n'est plus contrôlée, induisant soit un arrêt de la division cellulaire soit l'apoptose. En l'absence de p53, les cellules ont un génome instable et se transforment très rapidement. L'absence simultanée de p53 et mdm2 est viable. Cette viabilité indique que (1) mdm2 n'est pas indispensable à la division cellulaire mais (2) qu'elle est indispensable pour contrôler l'activité de p53. L'absence de cancer dans ces souris suggère donc que $\mathrm{mdm} 2$ pourrait avoir un rôle important dans l'apparition des tumeurs chez les animaux p53-/-. Néanmoins, cette observation est à prendre avec précaution car il faudra avoir plus de recul pour s'assurer de ce phénotype chez les animaux p53-/-; mdm2 -/-.

permis à ce jour de définir les causes de la dégénérescence. Ce résultat pourrait n'être que la énième inactivation de gène conduisant à des anomalies du développement, si les auteurs n'avaient eu l'idée de croiser les souris $m d m 2+/-$ avec des souris $p 53+/-[21,22]$. Les souris, issues de ces croisements, homozygotes pour la double délétion des gènes $m d m 2$ et $p 53$ ( $m d m 2-/-$; p53-/-) présentent de façon surprenante un développement embryonnaire normal et leur fréquence dans les portées est conforme à une transmission sans biais apparent (figure 4). Une autre surprise est que ces souris ne développent plus de tumeurs ( 7 mois de recul) contrairement aux souris parentales $p 53-/-$, indiquant que l'inactivation $\mathrm{du}$ gène $m d m 2$ compense celle du gène p53. L'hypothèse des auteurs pour expliquer la létalité des embryons $m d m 2-/-$ est simple: les fonctions de la protéine p53 n'étant plus réglées négativement par la protéine Mdm2, son accumulation dans la cellule induit, soit une apoptose, soit un arrêt de la division cellulaire, incompatibles avec un développement embryonnaire normal (ces deux mécanismes n'étant pas exclusifs). Lorsque l'on inactive le gène p53 dans cet environnement cellulaire, le cycle cellulaire peut reprendre de façon normale. L'absence de tumeurs chez les souris présentant cette double inactivation semble suggérer que la protéine Mdm2 est un partenaire essentiel dans l'apparition des tumeurs chez les souris p53-/- et qu'une dérégulation de l'expression de ce gène, en absence de p53, induirait un phénotype dominant nécessaire à la tumorigenèse.

\section{Coopération p53, Myc et Rb}

- $p 53$ et $c-M y c$

Dans le cas du gène c-myc, plusieurs travaux ont établi une augmentation du pouvoir transformant de cet oncogène dans un contexte de déficience en protéine p53. Le croisement de souris hétérozygotes $p 53+/-$ ou homozygotes $p 53-/-$ avec des souris surexprimant la protéine c-Myc sous contrôle du locus CD2 [23], du LTR de MMTV [24] ou du enhancer d'immunoglobuline $\mathrm{E} \mu$ [25], révèle en effet une nette augmentation de la lymphomagenèse consécutive à l'inactivation du gène p53, impliquant dans certains cas une inactivation fonctionnelle de l'allèle sauvage restant. La protéine c-Myc est un partenaire essentiel dans les processus de contrôle des voies de prolifération (c-Myc est impliquée dans l'expression de plusieurs gènes de cyclines et elle coopère avec la cycline $\mathrm{D} 1$ dans la transition G0/G1) et d'apoptose. Il semble que l'apoptose induite par c-Myc nécessite la présence de protéine p53 (m/s $n^{\circ} 9$, vol. 8, p. 1002) [26], des fibroblastes de souris p53-/- étant résistants à cette apotose (Tableau I). Dans un contexte de déficience en protéine p53, il devient de fait imaginable que l'accélération du processus de tumorigenèse passe par une abrogation de l'apoptose relayée par cMyc. La démonstration rapportée par Metz et al. [27] d'une immortalisation de cellules p53-/-d'origine hématopoiétique par un rétrovirus myc-raf, conforte cette hypothèse.

\section{- p53 et $R b$}

Les produits des gènes $p 53$ et $R b$ présentent, au sein de la cellule, des activités pour certaines similaires, puisque ces deux protéines nucléaires contrôlent de façon négative l'activité cyclique cellulaire $\left(m / s n^{\circ} 10\right.$, vol. 10, p. 1061) [28]. Ainsi la protéine $\mathrm{Rb}$ permet l'arrêt en phase G1 du cycle cellulaire par séquestration de facteurs de transcription de la famille E2F, facteurs eux-mêmes responsables de l'activation de gènes impliqués dans la réplication de l'ADN $\left(m / s n^{\circ} 9\right.$, vol. 11, p. 1358). L'inactivation par recombinaison homologue $\mathrm{du}$ gène $R b(R b-/-)$ se traduit par un développement abortif au $16^{\mathrm{e}}$ jour (Tableau I), les anomalies affectant majoritairement le tissu nerveux central et le tissu érythropoïétique [29-31]. Les souris hétérozygotes présentent, quant à elles, une claire prédisposition aux adénomes de l'hypophyse, selon un processus impliquant généralement une perte d'hétérozygotie [3]. Paradoxalement, alors que des altérations du gène $R b$ sont associées aux rétinoblastomes héréditaires et sporadiques (et ont été à l'origine de la caractérisation de ce gène), les souris $R b+/-$ ne développent aucune lésion au niveau de la rétine. Le croisement de souris hétérozygotes $R b+/-$ avec des souris déficientes pour un ou les deux allèles du gène $p 53$ a permis d'analyser l'incidence de la perte simultanée de 
ces deux gènes suppresseurs de tumeurs [32-34]. Plusieurs types de tumeurs décrites chez l'homme sont le siège de mutations dans les deux gènes $p 53$ et $R b$; par exemple, certains sarcomes, des carcinomes du poumon, du sein, du col de l'utérus, et du pancréas. Les résultats expérimentaux chez la souris ont permis d'établir un effet de coopération de l'inactivation de ces deux gènes au cours de la tumorigenèse, les tumeurs apparaissant beaucoup plus rapidement chez des souris $p 53+/-$; $R b+/-$ que chez les souris $p 53+/-$ ou chez les souris p53-/-; Rb+/- que chez les souris p53-/-. Bien que le spectre des tumeurs observées chez ces animaux diffère quelque peu suivant les travaux, il s'agit principalement de tumeurs d'origine endocrine, telles que des carcinomes des îlots de Langherans, des carcinomes médullaires de la thyroïde ou des tumeurs des glandes parathyroïdes, qui n'avaient jamais été observées chez les animaux ayant une simple altération. On retrouve également les adénomes hypophysaires, les sarcomes et lymphomes qui avaient déjà été décrits dans le cas des animaux déficients pour le gène $p 53$ uniquement. De nouvelles anomalies, telles que des pinéaloblastomes, des hyperplasies épithéliales bronchiques et des dysplasies de la rétine, ont aussi été retrouvées. De façon intéressante, certaines des tumeurs développées chez les souris $p 53+/-; R b+/-$ révèlent une perte d'hétérozygotie de l'un ( $R b$ ou $p 53$ ) ou des deux allèles sauvages $(R b$ et p53), soulignant le rôle important de l'inactivation de ces gènes dans le processus de transformation cellulaire. L'accélération notable de l'apparition des tumeurs chez les animaux doublement déficients renforce de plus l'idée d'un mécanisme «multi-étapes» de la cancérogenèse, impliquant l'activation d'oncogène(s) et/ou la perte de fonction de gènes suppresseurs de tumeurs tels que $R b$ et $p 53$.

\section{Conclusion}

L'ensemble de ces travaux montre velle d'expérimentation, où les études réalisées in vivo vont nous permettre de mieux appréhender la coopération entre gènes. Dans le cas de la protéine p53 et de son implication dans les processus néoplasiques, les résultats rapportés ici posent, pour certains, plus de questions qu'ils n'apportent de réponses: pourquoi les souris WAF1-/- et p53-/- ; mdm2-/- ne développent-elles pas de tumeurs? Quels sont les mécanismes responsables de l'apparition des tumeurs chez les animaux p53-/-? Malgré l'incontestable intérêt des modèles de souris transgéniques, il faut pourtant garder à l'esprit que les mécanismes de transformation cellulaire sont différents chez les rongeurs et chez les primates et que ces modèles animaux ne sont en fin de compte que des... modèles

\section{RÉFÉRENCES}

1. Brandon EP, Idzerda RL, McKnight GS. Targeting the mouse genome: a compendium of knockouts (part I). Curr Biol 1995; 5: 625-34.

2. Ozturk M, Soussi T. La p53 dans tous ses états: compte rendu du 8 e symposium p53. médecine/sciences 1994; 10: 1021-3.

3. Thomas G. Dix ans de recherche sur les prédispositions génétiques au développement des tumeurs. médecine/sciences 1995; $11: 336-48$

4. Lavigueur A, Maltby V, Mock D, Rossant J, Pawson T, Bernstein A. High incidence of lung, bone, and lymphoid tumors in transgenic mice overexpressing mutant alleles of the p53 oncogene. Mol Cell Biol 1989; 9: 3982-91.

5. Malkin D, Li FP, Strong LC, Fraumeni JF, Nelson CE, Kim DH, et al. Germ line p53 mutations in a familial syndrome of breast cancer, sarcomas, and other neoplasms. Science $1990 ; 250$ : 1233-8.

6. Donehower LA, Harvey M, Slagle BL, Mcarthur MJ, Montgomery CA, Butel JS, et $a l$. Mice deficient for p53 are developmentally normal but susceptible to spontaneous tumours. Nature 1992; 356: 215-21.

7. Armstrong JF, Kaufman MH, Harrison DJ, Clarke AR. High-frequency development abnormalities in p53-deficiencies mice. Curr Biol 1995; 5 : 931-6.

8. Harvey M, Vogel H, Morris D, Bradley A, Bernstein A, Donehower LA. A mutant p53 transgene accelerates tumour development in heterozygous but not nullizygous p53 deficient mice. Nature Genet 1995 ; 9 : 305-11.
9. Lane D. p53, guardian of the genome. Nature 1992 ; 358 : 15-6.

10. Nishino H, Knoll A, Buettner VL, Frisk CS, Maruta Y, Haavik J, et al. p53 wild-type and p53 nullizygous big blue transgenic mice have similar frequencies and patterns of observed mutation in liver, spleen and brain. Oncogene 1995; 11 : 263-70.

11. Sands AT, Suraokar MB, Sanchez A, Marth JE, Donehower LA, Bradley A. p53 deficiency does not affect the accumulation of point mutations in a transgene target. Proc Natl Acad Sci USA 1995; 92: 8517-21.

12. Provost GS, Kretz PL, Hammer RT, Matthews CD, Rogers BJ, Lundbergs KS, et al. Transgenic systems for in vivo mutation analysis. Mut Res 1993; 288: 133-49.

13. El-Deiry WS, Tokino T, Velculescu VE, Levy DB, Parsons R, Trent JM, et al. WAF1, a potential mediator of p53 tumor suppression. Cell 1993; 75 : 817-25.

14. Darbon J, Fesquet D, Cavadore J. De nouveaux régulateurs du cycle cellulaire: les protéines modulatrices des complexes Cdkcyclines. médecine/sciences 1995; 11: 349-56.

15. Johnson M, Dimitrov D, Vojta PJ, Barrett JC, Noda A, Pereirasmith OM, et al. Evidence for a p53-independent pathway for upregulation of SDI1/CIP1/WAF1/p21 RNA in human cells. Mol Carcinogen 1994; 11 : 59-64.

16. Michieli P, Chedid M, Lin D, Pierce JH, Mercer WE, Givol D. Induction of WAF1/CIP1 by a p53-independent pathway. Cancer Res 1994; 54 : 3391-5.

17. Akashi M, Hachiya M, Osawa Y, Spirin K, Suzuki G, Koeffler HP. Irradiation induces WAF1 expression through a p53-independent pathway in KG-1 cells. J Biol Chem $1995 ; 270$ : 19181-7.

18. Deng CX, Zhang PM, Harper JW, Elledge SJ, Leder P. Mice lacking p21 (C) P1/WAF1) undergo normal development, but are defective in G1 checkpoint control. Cell 1995; 82: 675-84.

19. Harel L. Les propriétés multiples des protéines de liaison des IGF (insulin-like growth factors): inhibiteurs et activateurs de croissance. médecine/sciences $1996 ; 12$ : sous presse.

20. Wu XW, Bayle JH, Olson D, Levine AJ. The p53 MDM2 autoregulatory feedback loop. Gene Develop 1993 ; 7: 1126-32.

\section{Thierry Soussi Virginie Lacronique}

Inserm U. 301, 27, rue Juliette-Dodu, 75010 Paris, France.

\section{TIRÉS À PART}

\section{T. Soussi.}


21. Jones SN, Roe AE, Donnehower LA, Bradley A. Rescue of embryonic lethality in Mdm2 deficient mice by absence of p53. Nature 1995; 378: 206-8.

22. Montes de Oca Luna R, Wagner DS, Lozano G. Rescue of early embryonic lethality in mdm2-deficient mice by deletion of p53. Nature 1995; 378: 203-6.

23. Blyth K, Terry A, Ohara M, Baxter EW, Campbell M, Stewart M, et al. Synergy between a human c-myc transgene and p53 null genotype in murine thymic lymphomas: contrasting effects of homozvgous and heterozygous p53 loss. Oncogene 1995; 10 : 1717-23.

24. Elson A, Deng CX, Campostorres J, Donehower LA, Leder P. The MMTV/c-myc transgene and p53 null alleles collaborate to induce T-cell lymphomas, but not mammary carcinomas in transgenic mice. Oncogene 1995 ; 11 : 181-90.

25. Hsu B, Marin MC, Elnaggar AK, Stephens LC, Brisbay S, McDonnell TJ. Evidence that c-myc mediated apoptosis does not require wild-type p53 during lymphomagenesis. Oncogene 1995 ; 11 : 175-9.

26. Hermeking H, Eick D. Mediation of cmyc-induced apoptosis by p53. Science 1994; 265: 2091-3.

27. Metz T, Harris AW, Adams JM. Absence of p53 allows direct immortalization of hematopoietic cells by the myc and raf oncogenes. Cell 1995; 82: 29-36.

28. Weinberg RA. The retinoblastoma protein and cell cycle control. Cell 1995; 81: 323-30.

29. Lee EYHP, Chang CY, Hu N, Wang YCJ, Lai CC, Herrup K, et al. Mice deficient for $\mathrm{Rb}$ are non viable and show defects in neurogenesis and haematopoiesis. Nature 1992; 359: 288-94.

30. Clarke AR, Maandag ER, van Roon M, van der Lugt NM, van der Valk M, Hooper $\mathrm{ML}$, et al. Requirement for a functional $\mathrm{Rb}-$ 1 gene in murine development. Nature 1992; 359: 328-30.

31. Jacks T, Fazeli A, Schmitt EM, Bronson RT, Goodell MA, Weinberg RA. Effects of an $\mathrm{Rb}$ mutation in the mouse. Nature 1992; 359: 295-300.

32. Williams BO, Remington L, Albert DM, Mukai S, Bronson RT, Jacks T. Cooperative tumorigenic effects of germline mutations in $\mathrm{Rb}$ and p53. Nature Genet 1994; 7: 480-4.

33. Morgenbesser SD, Williams BO, Jacks T, Depinho RA. p53-dependent apoptosis produced by Rb-deficiency in the developing mouse lens. Nature 1994; 371 : 72-4.

34. Harvey M, Vogel H, Lee EYHP, Bradley A, Donehower LA. Mice deficient in both p53 and rb develop tumors primarily of endocrine origin. Cancer Res 1995 ; 55 : 1146-51.

35. Martin K, Trouche D, Hagemeier C, Sorensen TS, Lathangue NB, Kouzarides T. Stimulation of E2F1/DP1 transcriptional activity by MDM2 oncoprotein. Nature 1995; 375: 691-4

36. Xiao ZX, Chen JD, Levine AJ, Modjtahedi $\mathrm{N}$, Xing J, Sellers WR, et al. Interaction between the retinoblastoma protein and the oncoprotein MDM2. Nature 1995; 375: 694-8. 